36. KARLIN, J. B., JUHN, D. J., STARR, J. I., SCANU, A. M., RUBENSTEIN, A. H.: Measurement of human high density lipoprotein apolipoprotein A-1 in serum by radioimmunoassay. J. Lipid Res. 30-37 (1976).

37. ASSMANN, G., SMOOTZ. E., ADLER, K., CAPURSO, A., OETTE, K.: The lipoprotein abnormality in Tangier Disease. Quantitation of $A$ apoproteins. J. clin. Invest. 59 564-575 (1977).

38. ALAUPOVIC, P.; CURRY, M. D., MCCONATHY, W. J.: Quantitative determina tion of human plasma apolipoproteins by electroimmunoassays. In: International Conrerence on Atherosclerosis. L. A. Carlson ed., p. 109-115. Raven Press, New York 1978. 39. SNIDERMAN, A., SHAPIRO, S., MARPOLE, D., SKINNER, B., TENG, B., KWITEROVICH, P. O.: Association of coronary atherosclerosis with hyperapobetalipoproteinemia (increased protein but normal cholesterol levels in human plasma low density ( $\beta$ ) lipoproteins). Proc. Natl. Acad. Sci. USA $77604-608$ (1980).

40. RIESEN, W. F., MORDASINI, R., SALZMANN, C., THELER, A., GURTNER, H. P. Apoproteins and lipids as discriminators of severity of coronary heart disease. Atherosclerosis 37 157-162 (1980)

41. UTERMANN, G., ALBRECHT, G., STEINMETZ, A.: Polymorphism of apolipoprotein E. I. Methodological aspects and diagnosis of hyperlipoproteinemia type III without ultracentrifugation. Clin. Genetics 14 351-358 (1978).

42. SCHONFELD, G., BAILEY, A., STEELMAN, R.: Plasma, apolipoprotein A-I and A-Il levels in hyperlipidemia. Lipids 13 951-959 (1978).

43. KUSHWAHA, R. S., HAZZARD, W. R., WAHL. P.W., HOOVER, J. J.: Type III hyperlipoproteinemia: Diagnosis in whole plasma by apolipoprotein $E$ immunoassay. Ann. Int. Med.: 86 509-516 (1977).
44. AVOGARO, P., CAZZOLATO, G., BITTOLO BON, G., QUINCI, G. B.: Are apolipoproteins better discriminators than lipids for atherosclerosis? Lancet I 901-903 (1979) 45. CHEUNG, M. C., ALBERS, J. J.: Measurement of apolipoprotein A-I and A-I levels in men and women by immunoassay. J. clin. Invest. 60 43-50 (1977).

46. RIESEN, W. F., MORDASINI, R. C.. MIDDELHOFF, G. W.: Quantitation of the two major apoproteins of human high density lipoproteins by solid phase radioimmunoassay. FEBS Letters 91 35-39 (1978).

47. WEISWEILER, P., SCHWANDT, P.: Lipid composition of serum lipoproteins in patients with primary type $\| \mathrm{b}$ and type IV hyperlipoproteinemia. Atherosclerosis 31 53-58 (1978).

Anschrift des Verfassers:

PD Dr. W. Riesen

Institut für klinisch-experimentelle Tumorforschung

der Universität Bern

Tiefenauspital

CH-3004 Bern, Schweiz.

\section{Referate aus Zeitschriften}

\section{Das Symptom - der klinisch-chemische Befund}

\section{K. Rommelt}

Department für Klinische Chemie des Zentrums

für Interdisziplinäre Medizinische Einheiten der Universität UIm Die Medizinische Welt 29, 1306, 1978

In dieser Arbeit sind alle Aufgabenstellungen zusammengefaßt, die im Verlauf einer Laboratoriumsuntersuchung anfallen, wobei die Prüfverfahren besonders beschrieben werden. Es handelt sich um Feststellungen, die von einer ad hoc Kommission der Deutschen Gesellschaft für Klinische Chemie 1978 erarbeitet wurden.

Zu 1.

Zu den vorbereitenden Arbeiten zur Durchführung einer Analyse gehören:

Auswahl von Kenngrößen, Untersuchungsverfahren und Untersuchungsmaterial.

Unter diese Tätigkeiten fallen:

Auswahl des geeigneten Untersuchungsmaterials, Entscheidung über die Wahl der Methode unter den Gesichtspunkten Zuverlässigkeit, Wirtschaftlichkeit, klinische Fragestellung und vorliegendes Untersuchungsgut; Kenntnis von Störfaktoren und biologischen Einflußgrößen.

Beurteilung der eingehenden Untersuchungsaufträge auf formale und sachliche Richtigkeit.

Überwachung der sachgerechten Durchführung der Materialgewinnung durch Erarbeitung von Vorschriften und Spezialanweisungen: Aufklärung von Fehlern bei der Materialabnahme; soweit möglich auch Uberwachung der Materialgewinnung.

Herr Prof. Rommel ist in diesem Jahr in Marburg verstorben. Er war Mitglied wichtiger Kommissionen und Arbeitsgremien. Dieses Referat gibt einen Ausschnitt aus der Arbeit einer solchen Kommission wieder.
Sicherung des Untersuchungsmaterials durch Überwachung des sachgerechten Transports und der sachgerechten Verwahrung des Materials; Identitätsprüfung von Untersuchungsanforderung und -material.

\section{Zu 2.}

Kontrolle und Überwachung der Durchführung der Analyse. Hierzu gehören folgende Tätigkeiten:

Adäquate Vorbereitung der Analyse, z. B. bei besonderem Untersuchungsmaterial (lipämisch, hämolytisch) und bei Vorliegen besonderer Störungsquellen.

Entscheidung über den Einsatz von Methoden und Geräten.

\section{Zu3.}

Beurteilung (analytische)

Hierunter versteht man die Bewertung der Analysendaten auf Grund der Ergebnisse der statistischen Qualitätskontrolle, der Wiederholungen u.a.

\section{Zu 4.}

Beurteilung (medizinische)

Diese Beurteilung wird ebenfalls im Laboratorium durchgeführt. Die Verfasser teilen diese Aufgabe in die folgenden Abschnitte: Plausibilitätskontrolle - ergänzende Untersuchungen - Vergleich mit den Vorwerten und dem Referenzkollektiv und Beurteilung von Befundmustern.

Abschließend stellen die Verfasser fest, daß der im Laboratorium erstelle Befund nicht eine einfache Feststellung von Sachverhalten mit technischen Mitteln ist, sondern eine analytische und synthetische Leistung aus Wissen und persönlicher Erfahrung. Für die Erstellung eines solchen Befunds sind nicht nur Kenntnisse der Durchführung und Eigenschaften der angewendeten analytischen Methoden und Instrumente, sondern auch der menschlichen Physiologie. Pathophysiologie und Pathobiochemie erforderlich, sowie Kenntnisse der Krankheitsbilder und deren Symptome. 